\title{
Perspectives of Geometric Analysis in PDEs
}

\author{
Steven G. Krantz ${ }^{1}$ - Vicentiu D. Radulescu ${ }^{2,3}$
}

Published online: 1 November 2019

(c) Mathematica Josephina, Inc. 2019

Functions, subvarieties, tensors, and other mathematical objects governed by natural partial differential equations provide usually deep insight into geometric structures. Geometric analysis is a discipline that strongly relies on the information provided by various classes of partial differential equations.

This special issue contains some contributions written by leading experts in problems at the interface between the geometric analysis and partial differential equations. Throughout, the main theme is to present the close interaction of these major research fields. More specifically, emphasis is placed on how the behavior of the solutions of a partial differential equation is affected by the geometry of the underlying manifold and vice versa.

V.R. would like to thank Professor Steven G. Krantz for giving him the opportunity to work together in this provocative editorial project.

Publisher's Note Springer Nature remains neutral with regard to jurisdictional claims in published maps and institutional affiliations.

Vicentiu D. Radulescu

vicentiu.radulescu@imar.ro

Steven G. Krantz

sgkrantz@gmail.com

1 Washington University in Saint Louis, Saint Louis, MO 63130, USA

2 Institute of Mathematics of the Romanian Academy, Bucharest, Romania

3 University of Craiova, 200585 Craiova, Romania 